\title{
Importancia de las plaquetas en las enfermedades cardiovasculares
}

\author{
Importance of platelets in cardiovascular diseases
}

\author{
"La medicina es la ciencia de la incertidumbre y el arte de la probabilidad" \\ sir William Osler, padre de la medicina interna
}

\begin{abstract}
L a fascinante historia de las plaquetas, aquellos pequeños fragmentos celulares que se desprenden del citoplasma de los megacariocitos, se remonta a los siglos XVII y XVIII [1]; no obstante, lo que hoy representan en el contexto de la salud y la enfermedad se inicia cuando sir William Osler (1849-1919), el padre de la medicina interna, las describe por primera vez en el año 1874 [2] y cuando Giulio Bizzozero (1846-1901) las caracteriza, en 1881, como un nuevo elemento forme de la sangre [3].
\end{abstract}

Con el correr del tiempo, y a partir de los primeros años del siglo $\mathrm{xx}$, las plaquetas se fueron posicionando como un parámetro más para incluir en el hemograma y, desde el punto de vista clínico, para definir y caracterizar algunas entidades hematológicas relacionadas con su recuento, como la púrpura trombocitopénica idiopática, hoy conocida como trombocitopenia autoinmune [4] y llamada, originalmente, enfermedad de Werlhof, que fue descrita en 1735 [5], muchísimos años antes de que se descubriesen las plaquetas y su papel en la hemostasia; al igual que aquellas asociadas a su morfología y funcionamiento, como el síndrome de Bernard-Soulier [6] y la trombastenia de Glanzmann [7], entre otras.

El desarrollo tecnológico relacionado con las plaquetas en la primera mitad del siglo XX se limitó a su recuento manual con cámaras desarrolladas para ese fin [8], y al tiempo de sangría [9] como única prueba de la función plaquetaria por muchos años; esta última aún ofertada en algunos laboratorios clínicos a pesar de ser una prueba obsoleta de acuerdo a lo definido claramente por el CAP (del inglés, College of American Pathology) y la ASCP (del inglés, American Society for Clinical Pathology) [10]. Todo lo anterior es suficiente para entender por qué el papel de las plaquetas fue considerado, por muchos años, como secundario.

Dos inventos revolucionaron la hematología, en general, y en el hemogramalos parámetros plaquetarios, en particular:

- El primero se desarrolló en 1953 y es conocido como principio de Coulter, el cual se define como un método que utiliza un campo eléctrico para contar y dimensionar partículas que se encuentran en suspensión en el líquido conductor, como reza en su patente [11], y que hoy se aplica en todos los contadores electrónicos de hematología y en las citometrías de flujo. Este principio revolucionó la hematología en general y, en el caso particular de las plaquetas, no solo mejoró su recuento, que antes se hacía por métodos manuales, sino que introdujo nuevos parámetros plaquetarios de utilidad clínica en el hemograma de rutina, como el volumen medio plaquetario, el ancho de distribución de las plaquetas, el plaquetocrito y el índice de plaquetas inmaduras o plaquetas reticuladas [12].

- El segundo se dio en 1963, la invención de la agregometría [13], con la cual se ha logrado estudiar el funcionamiento de las plaquetas y es el punto de partida del 
control de la terapia antiagregante; además, a partir de ella se han desarrollado nuevas tecnologías alrededor del tema, como la agregometría de impedancia, el PFA$100^{\circledR}[14]$, el VerifyNow ${ }^{\circledR}$ [15] y la tromboelastografía [16], entre otras, como métodos alternativos en el diagnóstico de las alteraciones de la función plaquetaria y en el manejo de la terapia centrada en la antiagregación plaquetaria.

Gracias al desarrollo tecnológico derivado de estos inventos hoy se han descubierto la mayoría de los secretos que guardan estos pequeños fragmentos celulares, lo que, a su vez, ha traído cambios que han impactado en el conocimiento y la práctica médica, entre los cuales vale la pena destacar los siguientes:

Se pasó de la clásica teoría de la cascada de la coagulación de MacFarlane [17], vigente por muchos años, en donde la coagulación está regulada exclusivamente por una cascada de activación de factores solubles (de la coagulación) en dos vías independientes, como se esquematiza en la figura 1, al modelo de la coagulación basado en la célula [18], en el cual la coagulación se activa mediante la interacción de las superficies celulares (el endotelio y las plaquetas), el factor tisular y el factor VII en tres fases simultáneas, conocidas como fase de iniciación, fase de amplificación y fase de propagación, como se esquematiza en la figura 2.

- Se pasó del enfoque de la prevención primaria y secundaria centrada en los cambios del comportamiento, por ejemplo, en la dieta [19], en la actividad física [20] y en dejar de fumar [21], así como en mejorar la gestión de los factores de riesgo, por ejemplo, mediante el buen control de la diabetes [22], de la hipertensión arterial [23], de la obesidad [24][25] y de los lípidos [26][27], entre otras medidas, a un enfoque más efectivo, centrado en los mismos objetivos pero con un nuevo componente que ha demostrado ser costo-efectivo y el eje central de la prevención primaria y secundaria: la antiagregación plaquetaria [28][29][30].

- Se pasó del control de la antiagregación plaquetaria centrado en el seguimiento clínico al control centrado en el seguimiento a través de pruebas de función plaquetaria por el laboratorio, lo que en la actualidad se conoce como un tratamiento personalizado [31], definido como «la utilización del fármaco adecuado para la persona indicada en el momento oportuno".

En el presente número de MEDICINA \& LABORATORIO se entrega el primero de dos módulos sobre la antiagregación con inhibidores del receptor plaquetario $\mathrm{P}_{2} \mathrm{Y}_{12}$, que complementan un módulo anterior sobre este tema relacionado con la resistencia a la aspirina [32]. En este primer módulo se analizará la fisiología plaquetaria a la luz de los conocimientos actuales y se presentarán los seis inhibidores del receptor plaquetario $\mathrm{P}_{2} \mathrm{Y}_{12}$ disponibles para su uso clínico y en vía de desarrollo para su uso a futuro.

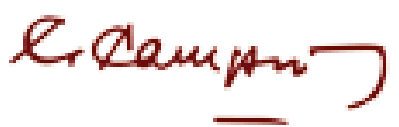

\section{Germán Campuzano Maya, MD}

Médico, especialista en Hematología y Patología Clínica

Director, Laboratorio Clínico Hematológico

Editor, Medicina \& Laboratorio

Medellín, Colombia, febrero 2017 
Via de activación por contacto (intrinseca) Via del factor tisular (extrinseca)

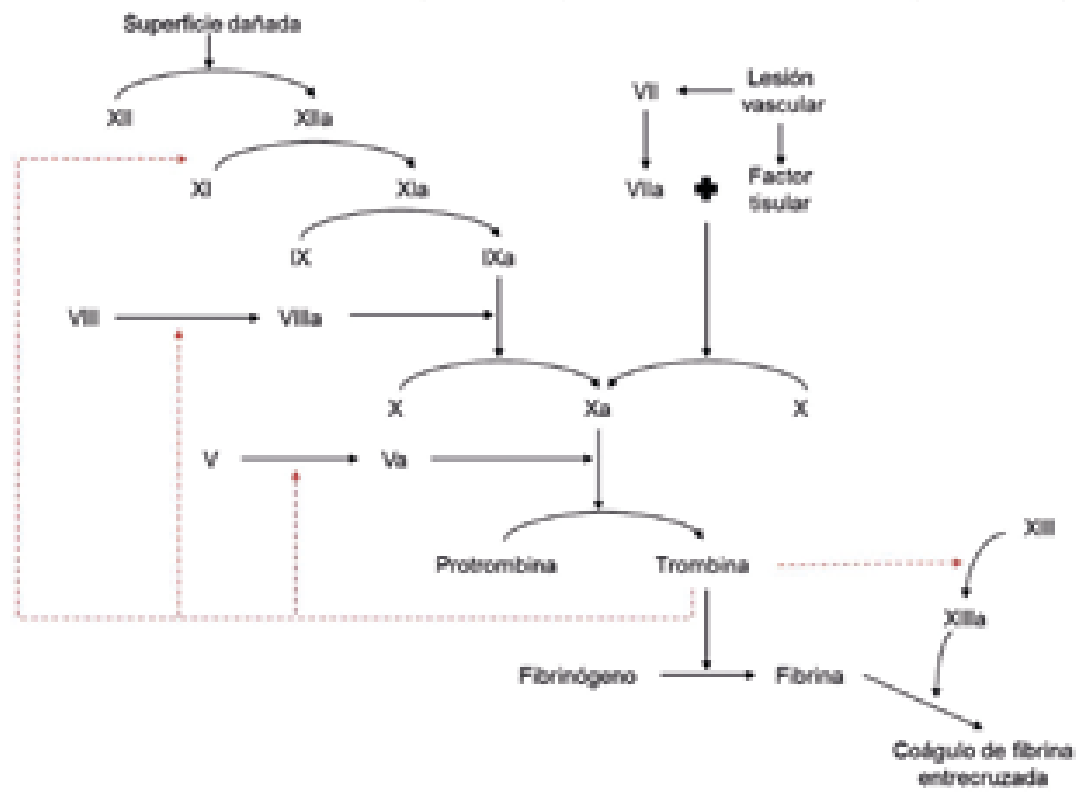

Figura 1. Cascada clásica de la coagulación sanguínea de acuerdo con MacFarlaine [17].

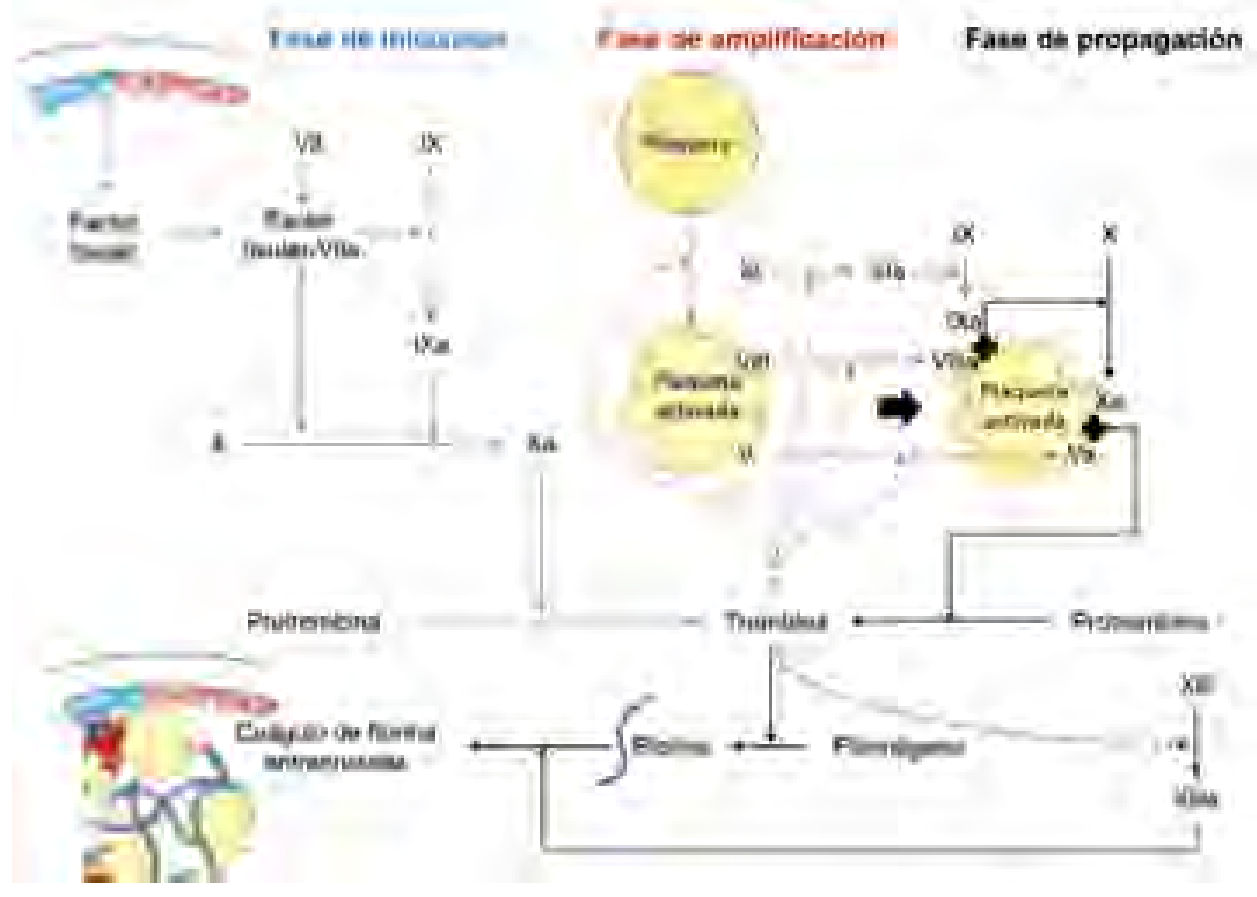

Figura 2. Modelo de coagulación basado en la célula [18]. 


\section{Bibliografía}

1. Robb-Smith $\mathbf{A H}$. Why the platelets were discovered. $\mathrm{Br} \mathrm{J}$ Haematol 1967;13:618-637.

2. Osler $\mathbf{W}$. An account of certain organisms occurring in the liquor sanguinis. Proc R Soc Lond 1874;22:3918.

3. Bizzozero G. Su di un nuovo elemento morfologico del sangue dei mammiferi e della sua importanza nella trombosi e nella coagulazione. L'Osservatore 1881;17:785-787.

4. Rodeghiero F, Stasi R, Gernsheimer T, Michel M, Provan D, Arnold DM, et al. Standardization of terminology, definitions and outcome criteria in immune thrombocytopenic purpura of adults and children: report from an international working group. Blood 2009;113:2386-2393.

5. Werlhof PG. De Variolis et Anthracibus. Hannover. 1735.

6. Bernard J, Soulier JP. Sur une nouvelle variété de dystrophie thrombocytairehémorragipare congénitale. Sem Hop 1948;24:3217-3223.

7. Glanzmann E. Hereditare hamorrhagische thrombasthenie. Ein Beitrag zur Pathologie der Blutplattchen. J Kinderkranken 1918;88:113.

8. Verso ML. Some nineteenth century pioners of haematology. Read at a Meeting in Melbourne of the Section of Medical History in the Australian Medical Association (Victorian Branch) on 9 June 1969.

9. Duke WW. The relation of blood platelets to hemorrhagic disease: Description of a method for determining the bleeding time and coagulation time and report of three cases of hemorrhagic disease relieved by transfusion. JAMA Cardiol 1910;55:1185-1192.

10. Peterson P, Hayes TE, Arkin CF, Bovill EG, Fairweather RB, Rock WA, Jr., et al. The preoperative bleeding time test lacks clinical benefit: College of American Pathologists' and American Society of Clinical Pathologists' position article. Arch Surg 1998;133:134-139.

11. Coulter WH. US Patent \#2,656,508. 1953.

12. Campuzano-Maya G. Del hemograma manual al hemograma de cuarta generación. Medicina \& Laboratorio 2007;13:551-550.

13. Born GV, Cross MJ. The aggregation of blood platelets. J Physiol 1963;168:178-195.

14. Campuzano-Maya G. PFA-100: una nueva prueba de función plaquetaria sustituta del tiempo de sangría. Medicina \& Laboratorio 2013;19 411-448.

15. Malinin A, Pokov A, Swaim L, Kotob M, Serebruany V. Validation of a VerifyNow-P2Y12 cartridge for monitoring platelet inhibition with clopidogrel. Methods Find Exp Clin Pharmacol 2006;28:315-322.

16. Yang $\mathbf{H}$, Li $\mathbf{Y}$, Jiang $\mathbf{Y}$, Lv $\mathbf{X}$. Thromboelastography for monitoring platelet function in unruptured intracranial aneurysm patients undergoing stent placement. Interv Neuroradiol 2015;21:61-68.

17. MacFarlane RG. An enzyme cascade in the blood clotting mechanism, and Its function as a biochemical amplifier. Nature 1964;202:498-499.

18. Hoffman M, Monroe DM, 3rd. A cell-based model of hemostasis. Thromb Haemost 2001;85:958-965.

19. Srinath Reddy K, Katan MB. Diet, nutrition and the prevention of hypertension and cardiovascular diseases. Public Health Nutr 2004;7:167-186.
20. Thompson PD, Buchner D, Pina IL, Balady GJ, Williams MA, Marcus BH, et al. Exercise and physical activity in the prevention and treatment of atherosclerotic cardiovascular disease: a statement from the Council on Clinical Cardiology (Subcommittee on Exercise, Rehabilitation, and Prevention) and the Council on Nutrition, Physical Activity, and Metabolism (Subcommittee on Physical Activity). Circulation 2003;107:3109-3116.

21. Rigotti NA, Pasternak RC. Cigarette smoking and coronary heart disease: risks and management. Cardiol Clin 1996;14:51-68.

22. Stratton IM, Adler Al, Neil HA, Matthews DR, Manley $\mathrm{SE}$, Cull CA, et al. Association of glycaemia with macrovascular and microvascular complications of type 2 diabetes (UKPDS 35): prospective observational study. BMJ 2000;321:405-412.

23. Chobanian AV, Bakris GL, Black HR, Cushman WC, Green LA, Izzo JL, Jr., et al. Seventh report of the Joint National Committee on Prevention, Detection, Evaluation, and Treatment of High Blood Pressure. Hypertension 2003;42:1206-1252.

24. Lavie CJ, McAuley PA, Church TS, Milani RV, Blair SN. Obesity and cardiovascular diseases: implications regarding fitness, fatness, and severity in the obesity paradox. J Am Coll Cardiol 2014;63:1345-1354.

25. Miner SE, Nield LE. Obesity cardiovascular disease and the failure of public health education. J Am Coll Cardiol 2016;67:2315-2316.

26. National Cholesterol Education Program Expert Pane on Detection E, Treatment of High Blood Cholesterol in A. Third Report of the National Cholesterol Education Program (NCEP) Expert Panel on Detection, Evaluation, and Treatment of High Blood Cholesterol in Adults (Adult Treatment Panel III) final report. Circulation 2002;106:3143-3421.

27. Gluckman TJ, Baranowski B, Ashen MD, Henrikson CA, McAllister M, Braunstein JB, et al. A practical and evidence-based approach to cardiovascular disease risk reduction. Arch Intern Med 2004;164:1490-1500.

28. The Medical Research Council's General Practice Research Framework. Thrombosis prevention trial: randomised trial of low-intensity oral anticoagulation with warfarin and low-dose aspirin in the primary prevention of ischaemic heart disease in men at increased risk. Lancet 1998;351:233-241.

29. De Gaetano G, Collaborative Group of the Primary Prevention $\mathbf{P}$. Low-dose aspirin and vitamin $\mathrm{E}$ in people at cardiovascular risk: a randomised trial in general practice. Collaborative Group of the Primary Prevention Project. Lancet 2001;357:89-95.

30. Antithrombotic Trialists Collaboration. Collaborative meta-analysis of randomised trials of antiplatelet therapy for prevention of death, myocardial infarction, and stroke in high risk patients. BMJ 2002;324:71-86.

31. Tantry US, Etherington A, Bliden KP, Gurbel PA. Antiplatelet therapy: current strategies and future trends. Future Cardiol 2006;2:343-366.

32. Campuzano-Maya G. Resistencia a la aspirina: un problema letante de alto riesgo. . Medicina \& Laboratorio 2016;22:411-448. 